\title{
Collaboration in BIM-based construction networks: A qualitative model of influential factors
}

\author{
Mehran Oraee, M. Reza Hosseini, David Edwards, Eleni Papadonikolaki
}

Engineering, Construction and Architectural Management

\begin{abstract}
Purpose - The purpose of this paper is to present a modified model for collaboration in BIM-based construction networks (BbCNs). Though BIM is increasingly adopted and implemented across the construction industry, the problems associated with the lack of collaboration among teams in BbCNs remains a major hindrance to reaping the full potential of BIM. Previous studies have been conceptual in nature. This paper, therefore, attempts to modify and validate existing conceptual models that describe collaboration in BbCNs.
\end{abstract}

Design/methodology/approach - To modify the conceptual model for collaboration in BbCNs, qualitative data through semi-structured interviews with BIM experts in the industry were collected and analysed using qualitative methods including the use of NVivo software.

Findings - The proposed model includes influential factors and their sub-factors to collaboration in $\mathrm{BbCNs}$, as well as considering their indicators. Findings reveal that several overlooked concepts, particularly unfavourable BIM contractual arrangements, act as the root causes of the unwillingness of team members in $\mathrm{BbCNs}$ to engage in collaborative efforts.

Research limitations - The findings of the study must be viewed in light of a few limitations. Firstly, the interviewees in this study were based in Australia, hence their perceptions of BIM collaboration are reflective of the sociotechnical setting of BIM-enabled projects in Australia. Also, the findings are based on the perception of experts in the field, rather than analysis of performance measures or quantitative assessment of associations among collaboration outcomes and various factors. This, however, provides the field with fertile grounds for future research.

Practical implications - The study benefits researchers by shifting the collaboration discourse in BIM-enabled projects from technology-related issues to the people and contractual-related domains. Moreover, the developed qualitative model provides industry professionals with a point of reference to improve collaboration on BIM-enabled projects. 
Oraee, M., Hosseini, M.R., Edwards, D. and Papadonikolaki, E. (2021), "Collaboration in BIM-based construction networks: a qualitative model of influential factors", Engineering, Construction and Architectural Management, Vol. ahead-of-print No. ahead-of-print. https://doi.org/10.1108/ECAM-10-2020-0865

Originality/value - Arguments provided in this study highlight the necessity of considering the contractual arrangement of BIM-related projects and foster the willingness of team members to collaborate. This can be addressed through the use of clear and comprehensive BIM Execution Plans and clearly explain the role of BIM managers in the process.

Keywords - Building Information Modelling; Coordination; Barrier; Interview; Contract

Paper type - Research paper 
Oraee, M., Hosseini, M.R., Edwards, D. and Papadonikolaki, E. (2021), "Collaboration in BIM-based construction networks: a qualitative model of influential factors", Engineering, Construction and Architectural Management, Vol. ahead-of-print No. ahead-of-print. https://doi.org/10.1108/ECAM-10-2020-0865

\section{Introduction}

Building Information Modelling (BIM) is the future trend, a relatively new disruptive innovation for the construction industry (Tulubas Gokuc and Arditi, 2017). The pervasiveness of BIM is exponentially on the rise (Liao and Teo, 2018). The global BIM market is projected to grow to $\$ 11.7$ billion by 2022, a compound annual growth rate (CAGR) of $21.6 \%$ during the forecast period $2016-$ 2022 (Lanjudkar, 2016). This presents industry with challenges on multiple fronts, not least of which is the necessity to conform to new contractual, behavioural and organisational norms within BIMrelated inter-organisational units (Poirier et al., 2017, Elghaish et al., 2019).

The major inter-organisational units utilised on current BIM-enabled projects are BIM-based Construction Networks (BbCNs), namely teams put together from experts of several specialist organisations to deliver BIM-related tasks (Oraee et al., 2017b). Collaboration among members of $\mathrm{BbCNs}$ is recognised as the key to success for BIM-enabled projects (Ashcraft, 2008, 2017). Nevertheless, this collaboration is still a serious challenge that affects BIM-enabled projects (Mignone et al., 2016, Merschbrock et al., 2018, Elghaish et al., 2019). Evidence obtained from industry acknowledges this challenge. According to the UK BIM Alliance, "it is the need for closer collaboration that's holding us back." (Kemp, 2017). There remain significant legal barriers, conflicts of interests and business considerations that make practitioners shy away from open collaborative processes on BIM-enabled projects (Liu et al., 2016b).

With the above in mind, there have been various attempts to address the issue of collaboration in BbCNs (Oraee et al., 2017b). A review of the literature shows that many scholars have tried developing innovative technologies to facilitate collaboration (Sacks et al., 2018, Elghaish et al., 2019). However, research on collaboration in BbCNs is dominated by the technology discourse (Oraee et al., 2017a), focusing on the software-central view of BIM (Papadonikolaki et al., 2019) and overlooking the crucial role of people management (Liu et al., 2017), despite the observation that people management is the major source of ineffective collaboration in BbCNs (Liao and Teo, 2018, Merschbrock et al., 2018). Scholars such as Mignone et al. (2016), Merschbrock et al. (2018), Li et al. (2019) emphasise the role of people, exploring collaboration issues in particular projects and discussing the solutions adopted, but their findings lack the ability to be generalized beyond the immediate settings of their studies. Besides, existing studies tend to focus on narrow areas associated with collaboration in $\mathrm{BbCNs}$, missing the bigger picture; they succeed in providing knowledge on individual trees, but fall short of offering a broader picture of the forest. As a result, despite the value added to the literature by existing studies, there is still little understanding of the nature and variety of the barriers that hinder collaboration in BbCNs (Oraee et al., 2017b). 
Oraee, M., Hosseini, M.R., Edwards, D. and Papadonikolaki, E. (2021), "Collaboration in BIM-based construction networks: a qualitative model of influential factors", Engineering, Construction and Architectural Management, Vol. ahead-of-print No. ahead-of-print. https://doi.org/10.1108/ECAM-10-2020-0865

The work by Oraee et al. (2019) is most applicable to the topic, in which a conceptual model of different barriers and influential factors to collaboration in BbCNs is presented. However, Oraee et al. (2019) do not go beyond the theoretical realm, in presenting the findings of their study which is based on a literature review. Their conceptual model of collaboration in $\mathrm{BbCNs}$ hence remains in need of validation and contextualisation in exposure to empirical data. Addressing this gap in the literature is the raison d'être for the present study, which extends the work conducted by Oraee et al. (2019), by offering empirical validation of the list of factors and barriers provided by exposure to the scrutiny of experts in the field.

\section{Background}

\subsection{Collaboration in the construction sector}

From a behavioural science perspective, Wood and Gray (1991, p. 146) discuss that: "collaboration occurs when a group of autonomous stakeholders of a problem domain engages in an interactive process, using shared rules, norms, and structures, to act or decide on issues related to that domain." In the construction context, collaboration describes all situations where various parties work together, for example partnering, alliancing, joint ventures or networking (Hughes et al., 2012). Collaboration in construction therefore largely falls within the procurement discourse (Durdyev et al., 2019, Elghaish et al., 2019). That is, collaboration occurs by adopting delivery methods that establish collaborative arrangements in delivering projects (Kuiper and Holzer, 2013, Hughes et al., 2015). One effective solution - for enhancing collaboration in project delivery - is through adopting technologies that provide project team members with a single shared interface and Common Data Environment (CDE) (Merschbrock and Munkvold, 2015, Charef et al., 2019). The solution relies on using data exchange frameworks and technological innovations that facilitate collaboration (Alreshidi et al., 2017). In response, BIM has emerged as an advanced methodology that provides the needed CDE and facilitates sharing of information and data among various disciplines (Kuiper and Holzer, 2013, Liao and Teo, 2018, Piroozfar et al., 2019), as discussed next.

\subsection{Building Information Modelling}

The concept of Building Information Modelling (BIM), as identified by the AECO sector in recent years, refers to a set of interacting processes and technologies that are used to integrate and manage essential construction project information in digital format throughout the project life cycle (Succar, 2009, Holzer, 2016). In this context, BIM refers to a technological innovation that provides interorganisational models and integrates various project stakeholders (Bryde et al., 2013, Garg, 2017); it provides one single point of reference for designers, engineers, contractors, facility managers and owners (Bryde et al., 2013, Sacks et al., 2018). BIM-enabled projects thus require construction professionals to collaborate frequently through a common information-sharing platform (Sackey et al., 
Oraee, M., Hosseini, M.R., Edwards, D. and Papadonikolaki, E. (2021), "Collaboration in BIM-based construction networks: a qualitative model of influential factors", Engineering, Construction and Architectural Management, Vol. ahead-of-print No. ahead-of-print. https://doi.org/10.1108/ECAM-10-2020-0865

2015, Oraee et al., 2019). As their main inter-organisational unit, BIM-enabled projects rely on BIMbased Construction Networks (BbCNs) (Oraee et al., 2017b). These comprise groups of specialist organisations contracted to execute BIM-related works (Grilo et al., 2013). In BbCNs, collaborative working is of crucial importance (Mignone et al., 2016). Indeed, enhancing collaboration in project delivery has been a major selling point of BIM (Liu et al., 2017). Collaboration defines the effectiveness and performance in delivering projects (Eastman, 2011, Sacks and Pikas, 2013) and the success of BIM-enabled projects relies on effective collaboration among stakeholders (Bassanino et al., 2014). However, the envisaged potential of BIM remains untapped in the absence of effective collaboration (Zhang et al., 2017, Merschbrock et al., 2018).

\subsection{Collaboration in BbCNs}

With the rise of BIM as the state-of-the-art technology to foster collaboration, BbCNs have become the centrepiece of collaboration on construction projects (Grilo et al., 2013, Liu et al., 2016a, Mignone et al., 2016). However, despite the rise of collaboration tools and technologies, collaboration in BbCNs is identified as an underperforming area. According to the study by (Mignone et al., 2016), poor collaboration in $\mathrm{BbCNs}$ has resulted in the ineffectiveness and lack of performance in project teams, eventually leading to design clashes, omissions and errors (Sackey et al., 2015). The existing literature shows that collaboration in BIM-based construction networks (BbCNs) is problematic and requires significant attention for collaboration to be fostered in BIM-enabled organisations and construction projects. In response to the low-performance collaboration capability of BIM, the research by McGrawHill Construction (2017) identified that "investment in developing collaborative processes with other parties" is in the top five forecast investments in BIM, as proposed by major stakeholders (architects, engineers and contractors) in the AECO sector. Moreover, the report focused on very high-level BIMengaged global contractors with the findings illustrating that "improving collaboration (both internally and externally) between project major stakeholders" is in the top five future BIM investment strategies for these contractors. Ashcraft (2008) proffered the view that a BIM-enabled project in the absence of collaboration is akin to merely "scratching the surface" in terms of realising the software's full capability. This highlighted the crucial role of access by major stakeholders to interoperable processes, software and packages for BbCNs (Grilo and Jardim-Goncalves, 2010, Hu et al., 2016).

\subsection{Enhancing collaboration in BbCNs}

As discussed, collaboration stands at the centre of BbCNs (Grilo et al., 2013, Mignone et al., 2016). Despite this, collaboration in BbCNs is a challenge (Sacks et al., 2018, Oraee et al., 2019); it remains an unresolved problem and the topic, therefore, forms one of the core clusters of BIM-related research (Durdyev et al., 2019, Lemaire et al., 2019, Oraee et al., 2019). 
Oraee, M., Hosseini, M.R., Edwards, D. and Papadonikolaki, E. (2021), "Collaboration in BIM-based construction networks: a qualitative model of influential factors", Engineering, Construction and Architectural Management, Vol. ahead-of-print No. ahead-of-print. https://doi.org/10.1108/ECAM-10-2020-0865

Previous studies on the topic have recommended various approaches to enhancing collaboration in $\mathrm{BbCNs}$. These include, for the major part, enhancing the functionality of technology and developing new technologies that facilitate collaborative working (Wang et al., 2014, Sacks et al., 2018, Elghaish et al., 2019). Moreover, developing data exchange protocols and working arrangements that support collaboration are other avenues explored (Kassem et al., 2014, Merschbrock and Munkvold, 2014, Elghaish et al., 2019). Indeed, focusing on technology to enhance collaboration has dominated research on the topic (Cao et al., 2016, Oraee et al., 2017a, Merschbrock et al., 2018), but research focus has overlooked the significant impacts of the people management components that influence collaboration in BbCNs, a point argued by Liu et al. (2017), Merschbrock et al. (2018) and Liao and Teo (2018), among others.

Scholars have thus covered a wide range of sociotechnical factors in addressing the issues of ineffective collaboration in BbCNs, focusing on the technology-oriented perspective, as well as human-computer interactions and people management (Jin et al., 2017, Matarneh et al., 2019). Nevertheless, as discussed, the extant literature on the topic suffers from various shortcomings, the result of which is that detailed knowledge of the nature and variety of the pool of factors and barriers that affect collaboration in BbCNs is yet to be acquired. This is discussed next.

\subsection{Summary and gap}

The failure of industry practitioners in shifting common practices to foster collaboration among members in BbCNs remained a still present and still vexing problem (Poirier et al., 2016, Oraee et al., 2017a, Alreshidi et al., 2018). Identifying the barriers that act as the root causes behind this problem is a predicate to provide remedial solutions (Merschbrock et al., 2018). And studies to address this need remain much needed in the related literature. To the best of the authors' knowledge, the only scholarly work available in the field is a conceptual model of barriers to collaboration in BbCNs developed by Oraee et al. (2019). Indeed, this conceptual model lacks validity and recognition from field experts, as the authors argue (cf.Oraee et al., 2019). This gap is the driving force behind conducting the present study, that is, the motivation is to modify and validate this published conceptual model through collecting empirical data via conducting interviews with BIM professionals in the industry. The conceptual model is described as follows.

\subsection{The conceptual model}

According to Oraee et al. (2019), factors that hinder collaboration in BbCNs are grouped within major constructs (Process, Actor, Context, Team and Task) and their sub-factors (as illustrated in Figure 1). 


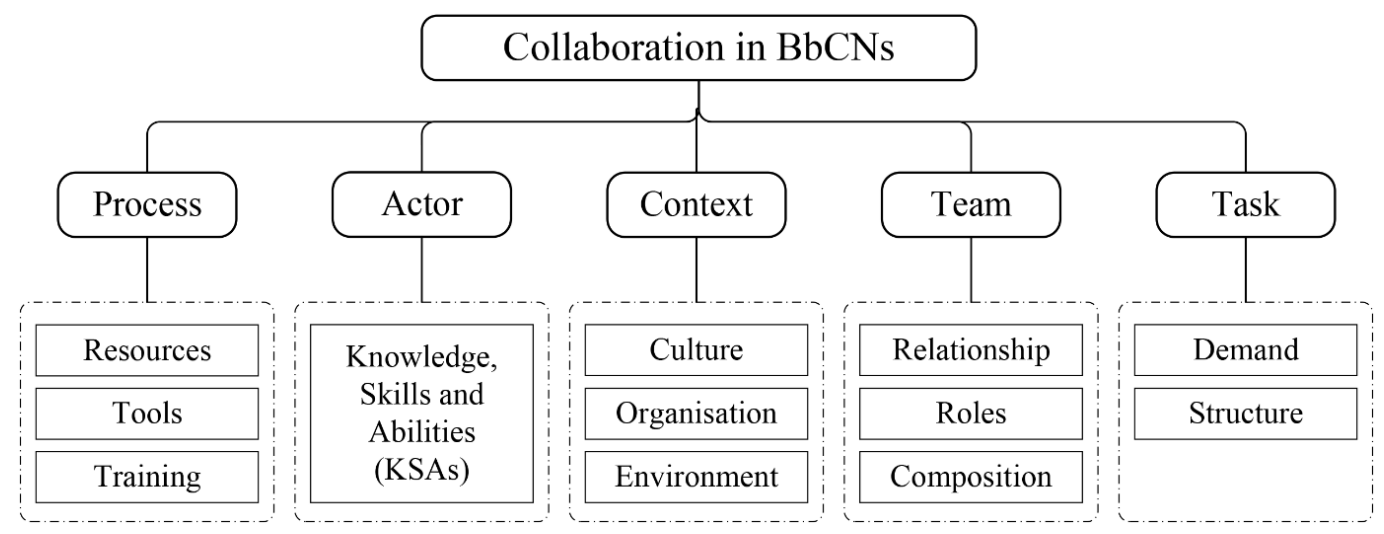

Figure 1: Conceptual Model for collaboration in BbCNs - adapted from Oraee et al. (2019)

The construct 'Process' refers mainly to the technical side of collaboration which includes resources and tools, as well as relevant training for collaboration. 'Actor' refers to the knowledge, skills and abilities (KSAs) in BIM that are required by people working in the process of collaboration in BIMenabled organisations and projects. 'Context' reflects the specific environment that all these identified factors are set within, whilst 'Team' reflects the roles and relationships between a group of actors from different disciplines and/or organisations working collaboratively to deliver BIM deliverables and eventually the project. Finally, 'Task' refers to the nature of BIM tasks including demand and their structure.

\section{Research methods}

\subsection{Research approach}

Collaboration in BbCNs is still a less explored area. As proposed by Neuman (2006) and Punch (2005), investigating a 'little-understood phenomenon' requires the deployment of qualitative techniques for collecting data from the field. To be specific, as argued by Ahmed et al. (2016), qualitative studies are best suited to careful explorations of the real-life practices and experiences of the actors involved in the topic of the study (Oraee et al., 2017b, Merschbrock et al., 2018). Given the emphasis of this study on validation through experts, a qualitative approach was deemed suitable. Conducting interviews is one of the most effective methods for undertaking a qualitative approach. As mentioned, this study aimed at presenting the conceptual model to subject matter experts and build confidence in the applicability and confidence in the model through conducting interviews with these experts. The aim was engaging in conversation with experts about the model, to modify, confirm and add/remove components. As argued by Andersen et al. (2012), this is a common approach by researchers, which can be used for verifying accurate models that present the nature of relationships and show interactions among various components like system dynamic models. In fact, using interviews to modify conceptual models is an acceptable practice in the literature (Black, 2002). Moreover, interviews can assist researchers to use 
Oraee, M., Hosseini, M.R., Edwards, D. and Papadonikolaki, E. (2021), "Collaboration in BIM-based construction networks: a qualitative model of influential factors", Engineering, Construction and Architectural Management, Vol. ahead-of-print No. ahead-of-print. https://doi.org/10.1108/ECAM-10-2020-0865

existing theories in facilitating the emergence of granular knowledge and validating existing knowledge using data from the context at hand (Creswell, 2014).

According to Creswell $(2014$, p. 65) "... the theory may appear at the beginning and be modified or adjusted based on participant views". This is the purpose of considering interviews as the qualitative method to be utilised in this study. Indeed, findings from interviews will assist the researcher to refine the framework on collaboration in BbCNs. As inferred by Rowley (2012, p. 262), "the most common type of interview is the semi-structured interview". Deploying semi-structured interviews in the present study was aimed at providing a basis for modifying the theoretical framework of the study on collaboration in BbCNs.

\subsection{Sampling}

Interviewees were selected using the 'purposive sampling,' namely, “... sampling in a deliberate way, with some purposes or focus in mind" (Punch, 2005, p. 187). Purposive sampling enables researchers to fulfil the research objectives and simultaneously use and control the level of variation among interviewees. Interviewees were therefore recruited by taking into consideration a trade-off between

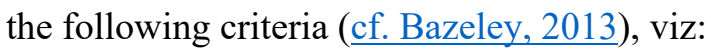

- Sample heterogeneity: Adequate variation among interviewees to allow for comparative analysis; and

- Sample homogeneity: Limited variation to allow for intensive study.

The trade-off was considered to balance the level of homogeneity among participants and ensure access to a sample of participants whose attributes are adequately heterogenous, as recommended by Robinson (2014). By maintaining a measure of sample homogeneity, data acquired from all participants will be deemed reliable and applicated within a defined setting, where all participants satisfy one common criterion: the possession of an adequate experience and knowledge on the key concepts related to the topic of the study. The rationale of selecting a reasonably heterogeneous sample is that any commonality found across participants is more likely to be a widely generalisable phenomenon than a commonality found in a homogenous group. Therefore, heterogeneity of sample helps provide evidence that findings are not solely reflective of one category of participants like designers. Having participants belonging to different groups and professional categories will assist in arguing that findings apply to various groups within the setting at hand (Robinson, 2014).

The number of interviewees was considered to be between three and 16, as a reasonable preliminary estimation for defining the sample size (Bazeley, 2013). Thus, an initial list of 54 BIM experts (potential interviewees) was identified, who had relevant expertise in BIM and considerable years of experience working in the Australian construction industry. It is noteworthy to mention that the number of BIM 
experts is constrained to a limited pool of practitioners. The list was prepared by targeting individual members of groups dedicated to BIM in social professional networks such as LinkedIn, recommended as a reliable source for assessing experts' qualifications (Julie et al., 2014). The concept of saturation must be considered for qualitative studies that use purposive sampling. Bazeley (2013) states that saturation is the point at which "no new information is being added to coding categories (data saturation) or to the emerging theories (theoretical saturation) through adding further cases to the analysis." The present study's protocol for recognising the point of saturation was the point at which after three interviews, no new code or theory emerges (Hosseini et al., 2016). As a result, interviews were stopped after conducting interviews with 11 interviewees (i.e. saturation point was reached after the 8th interview), whose profiles are detailed in Table 1. In terms of the adequacy of the number of interviewees, as discussed, generally there is a limited pool of experts in this field with reasonable experience in BIM and collaboration. Indeed, many self-claimed BIM experts (e.g. BIM drafters) only have knowledge and skills in developing models and may not be involved in the collaboration process. So, this further limits the pool of experts for data collection. In this research and during the interviews, most of the findings were found through the first 8 interviews. After these 8 interviews and through the next three interviews, no new code was found, showing a saturation in the collected data. As discussed by Bazeley (2013, p. 50), saturation can be reached with any number of interviewees and this could be between six and 12. As a result, given the limited pool of BIM experts, having 11 respondents in the present study provided a reasonable and acceptable sample size for collecting qualitative data.

Table 1. Interviewees' profiles

\begin{tabular}{|c|c|c|c|c|c|c|c|}
\hline \multirow[b]{2}{*}{ No } & \multirow[b]{2}{*}{ ID } & \multirow[b]{2}{*}{ Occupation } & \multicolumn{2}{|c|}{ Experience in } & \multirow[b]{2}{*}{ Organisation type } & \multirow[b]{2}{*}{ Project size } & \multirow{2}{*}{$\begin{array}{c}\text { Team's } \\
\text { geographical } \\
\text { spread }\end{array}$} \\
\hline & & & $\begin{array}{l}\text { Total } \\
\text { (Year) }\end{array}$ & $\begin{array}{c}\text { BIM } \\
\text { (Year) }\end{array}$ & & & \\
\hline 1 & $\mathrm{~A}$ & BIM MEP Manager & 16 & 3 & Building services & Large & Globally \\
\hline 2 & $\mathrm{~B}$ & BIM MEP Designer & 15 & 5 & $\mathrm{~A} / \mathrm{E}$ & Large & Australia \\
\hline 3 & $\mathrm{C}$ & BIM Consultant & 12 & 3 & Design & Small-Medium & Australia \\
\hline 4 & $\mathrm{D}$ & BIM Manager & 17 & 5 & Building services & Large & Australia \\
\hline 5 & $\mathrm{E}$ & BIM Leader & 17 & 8 & $\mathrm{GC}$ & Large & Australia \\
\hline 6 & $\mathrm{~F}$ & BIM Advocate & 18 & 9 & $\mathrm{~A} / \mathrm{E}$ & Large & Globally \\
\hline 7 & G & BIM Manager & 11 & 8 & Fire consultancy & Small-Medium & Australia \\
\hline 8 & $\mathrm{H}$ & BIM Consultant & 20 & 3 & Architectural & Small-Medium & Australia \\
\hline 9 & I & BIM Manager & 14 & 7 & CIR & Large & Globally \\
\hline 10 & $\mathrm{~J}$ & BIM Consultant & 27 & 4 & $\mathrm{GC}$ & Large & Melbourne \\
\hline 11 & $\mathrm{~K}$ & BIM Consultant & 16 & 8 & $\mathrm{~A} / \mathrm{E}$ & Large & Globally \\
\hline $\begin{array}{l}\text { A/E: } \\
\text { GC: } \\
\text { CIR: }\end{array}$ & $\begin{array}{l}\text { Archi } \\
\text { iener }\end{array}$ & $\begin{array}{l}\text { ural and Engineering } \\
\text { ontractor } \\
\text { ion, Infrastructure anc }\end{array}$ & estate & & & & \\
\hline
\end{tabular}


Table 1 illustrates that interviewees had a minimum of three years and a maximum of nine years of experience in working with BIM. The type of organisations they are working in are ranged from architectural and engineering $(\mathrm{A} / \mathrm{E})$ to general contractor $(\mathrm{GC})$, building services and infrastructure and real estate firms. The location was also incorporated in interviewee selection - in this case, Australia. For a number of interviewees, team members were dispersed across a city or a state in Australia while for others, team members were scattered across the globe and in different countries. Moreover, the size of each interviewee's employer organisation was also included in the selection criteria. As a result, the categorisation for construction organisations was thematically clustered into two dichotomous groupings of either small-medium or large-sized organisations. Thus, the sampling strategy in the current study was 'maximum variation', which is a subset of purposive sampling. As argued by Kitto et al. (2008, p. 244) “... maximum variation is the ideal when a holistic overview of the phenomenon is sought" - this is certainly the case for the present study.

\subsection{Data collection}

Of the 11 interviewees, two agreed to attend face-to-face interviews, whilst nine interviews were completed over the phone. An integral part of the adopted procedure for conducting each interview was for the interviewer to first explain to the interviewee the concept of collaboration in BIM-enabled construction projects and further elucidate upon the study's objectives. Then, once the researcher had ensured that the interviewee's interpretation of collaboration in BIM projects was consistent with the definition of collaboration in the present study, the main questions were posed and discussed. Each interview lasted around 45-60 minutes with the interview being audio recorded.

The interview sessions were mainly focused on modifying the conceptual model developed by Oraee et al. (2019), as illustrated in Figure 1, thus ensuring that it met the study objectives of being a practical model and applicable to the construction industry. During the interview sessions, interviewees were asked to discuss the challenges (barriers) in collaboration in BbCNs and add any factors and/or sub-factors that were not included in the conceptual model. This common application of qualitative research ensures that the research findings remain connected to the existing body of knowledge whilst simultaneously creating new knowledge (Bazeley, 2013).

\section{From interviews to model development}

In this study, NVivo (developed by QSR International Pty Ltd.) was utilised as a qualitative data analysis software tool, given its capabilities for providing in-depth insight into collected data and the benefits, it provides for indexing, marking and sorting qualitative data with greater speed, accuracy and flexibility (…Bazeley, 2013).

\subsection{Data analysis}


Coding is the process of assigning names, tags or labels to different pieces of unstructured data - in this case, transcripts from interviews (Punch, 2005). This study utilised a mixed approach that involved the integration of deductive and inductive coding: codes were developed in view of the existing literature (theory-driven codes) in tandem with interviewees' subjective views (data-driven codes). A well-established method for assigning either theory-driven or data-driven codes is to focus upon similarity, comparison and contrast against a theoretical structure (Bazeley, 2013). Thus, the theoretical structure - namely the conceptual model, illustrated in Figure 1 - was used to organise the emerging themes and codes. The conceptual model set the foundation for empirical fieldwork, during which collected qualitative data was interpreted and then coded against the relevant factors that hinder collaboration in BbCNs. Through this coding and analysis, factors in the conceptual model were either confirmed, modified or removed. Any new factors and sub-factors identified were created in addition to the confirmed or modified factors in the conceptual model. Figure 2 illustrates the process of comparing the conceptual model to the findings of the qualitative analysis in the present study. 


\section{University Library}

\section{- M M N E R VA A gateway to Melbourne's research publications}

Minerva Access is the Institutional Repository of The University of Melbourne

Author/s:

Oraee, M;Hosseini, MR;Edwards, D;Papadonikolaki, E

Title:

Collaboration in BIM-based construction networks: a qualitative model of influential factors

Date:

2021-04-07

Citation:

Oraee, M., Hosseini, M. R., Edwards, D. \& Papadonikolaki, E. (2021). Collaboration in BIMbased construction networks: a qualitative model of influential factors. ENGINEERING CONSTRUCTION AND ARCHITECTURAL MANAGEMENT, 29 (3), https://doi.org/10.1108/ ECAM-10-2020-0865.

Persistent Link:

http://hdl.handle.net/11343/273713 